\title{
BMJ Open Severity of respiratory failure at admission and in-hospital mortality in patients with COVID-19: a prospective observational multicentre study
}

\author{
Pierachille Santus (D , , ${ }^{1,2}$ Dejan Radovanovic, ${ }^{2}$ Laura Saderi, ${ }^{3}$ Pietro Marino, ${ }^{4}$ \\ Chiara Cogliati, ${ }^{5}$ Giuseppe De Filippis, ${ }^{6}$ Maurizio Rizzi, ${ }^{2}$ Elisa Franceschi, ${ }^{1,2}$ \\ Stefano Pini, ${ }^{1,2}$ Fabio Giuliani, ${ }^{7}$ Marta Del Medico, ${ }^{5}$ Gabriella Nucera, ${ }^{4}$ \\ Vincenzo Valenti, ${ }^{7}$ Francesco Tursi, ${ }^{8}$ Giovanni Sotgiu ${ }^{3}$
}

To cite: Santus $P$,

Radovanovic D, Saderi L, et al. Severity of respiratory failure at admission and in-hospital mortality in patients with COVID-19: a prospective observational multicentre study. BMJ Open 2020;10:e043651. doi:10.1136/ bmjopen-2020-043651

- Prepublication history and additional material for this paper is available online. To view these files, please visit the journal online (http://dx.doi.org/10. 1136/bmjopen-2020-043651).

PS and DR contributed equally.

PS and DR are joint first authors.

Received 11 August 2020

Revised 23 September 2020

Accepted 24 September 2020

Check for updates

(c) Author(s) (or their employer(s)) 2020. Re-use permitted under CC BY-NC. No commercial re-use. See rights and permissions. Published by BMJ.

For numbered affiliations see end of article.

Correspondence to Dr Pierachille Santus; pierachille.santus@unimi.it

\section{ABSTRACT}

Objectives COVID-19 causes lung parenchymal and endothelial damage that lead to hypoxic acute respiratory failure (hARF). The influence of hARF severity on patients' outcomes is still poorly understood.

Design Observational, prospective, multicentre study. Setting Three academic hospitals in Milan (Italy) involving three respiratory high dependency units and three general wards.

Participants Consecutive adult hospitalised patients with a virologically confirmed diagnosis of COVID-19. Patients aged $<18$ years or unable to provide informed consent were excluded.

Interventions Anthropometrical, clinical characteristics and blood biomarkers were assessed within the first 24 hours from admission. hARF was graded as follows: severe (partial pressure of oxygen to fraction of inspired oxygen ratio $(\mathrm{PaO} 2 / \mathrm{FiO2})<100 \mathrm{~mm} \mathrm{Hg})$; moderate $(\mathrm{PaO2} / \mathrm{FiO} 2$ 101-200 mm Hg); mild (Pa02/Fi02 201-300 mm Hg) and normal $(\mathrm{PaO} 2 / \mathrm{FiO} 2>300 \mathrm{~mm} \mathrm{Hg})$.

Primary and secondary outcome measures The primary outcome was the assessment of clinical characteristics and in-hospital mortality based on the severity of respiratory failure. Secondary outcomes were intubation rate and application of continuous positive airway pressure during hospital stay.

Results 412 patients were enrolled (280 males, 68\%). Median (IQR) age was 66 (55-76) years with a Pa02/ Fi02 at admission of 262 (140-343) mm Hg. 50.2\% had a cardiovascular disease. Prevalence of mild, moderate and severe hARF was $24.4 \%, 21.9 \%$ and $15.5 \%$, respectively. In-hospital mortality proportionally increased with increasing impairment of gas exchange $(p<0.001)$. The only independent risk factors for mortality were age $\geq 65$ years (HR 3.41; 95\% Cl 2.00 to 5.78, $\mathrm{p}<0.0001$ ), Pa02/ Fi02 ratio $\leq 200 \mathrm{~mm} \mathrm{Hg}$ (HR 3.57; 95\% Cl 2.20 to 5.77 , $\mathrm{p}<0.0001$ ) and respiratory failure at admission (HR 3.58; $95 \% \mathrm{Cl} 1.05$ to $12.18, \mathrm{p}=0.04$ ).

Conclusions A moderate-to-severe impairment in $\mathrm{PaO2} /$ Fi02 was independently associated with a threefold increase in risk of in-hospital mortality. Severity of respiratory failure is useful to identify patients at higher risk of mortality.

\section{Strengths and limitations of this study}

- This was a multicentre, prospective study.

- The study has enrolled a conspicuous number of well-characterised patients hospitalised with COVID-19 pneumonia.

- A selection bias may be due to the high number of severe patients due to the hub characteristics of the participating centres.

- Not all patients were evaluated in room air conditions at admittance, thus potentially underestimating the severity of the study sample.

Trial registration number NCT04307459

\section{INTRODUCTION}

SARS-CoV-2 and the related COVID-19 has caused a pandemic and $\sim 860000$ deaths worldwide. ${ }^{1}$ The clinical spectrum can range from mild symptoms (eg, fever and malaise) to severe hypoxic respiratory failure, sepsis, multiorgan involvement and death. The infection appears to induce an inflammatory reaction with pulmonary infiltrates generating hypoxaemia secondary to intraparenchymal shunt and ventilation/perfusion mismatch, favoured by endothelial damage and dysfunction, and altered regulation of perfusion and associated with macroembolism and/or microembolism. ${ }^{2}{ }^{3}$ So far, risk factors such as older age ${ }^{4-6}$ severity of clinical presentation, ${ }^{4-7}$ increased D-dimer values, ${ }^{4}$ cardiovascular disease (CVD) ${ }^{45}$ and hypertension $^{5-8}$ have been associated with unfavourable outcomes.

It has been proposed that clinical severity of COVID-19 should depend on the presence of any of the following criteria: a partial pressure of oxygen to fraction of inspired oxygen 
$(\mathrm{PaO} 2 / \mathrm{FiO} 2)$ ratio $<300 \mathrm{~mm} \mathrm{Hg}$, a respiratory rate $>30$ per min and a peripheral oxygen saturation (SpO2) $<93 \%{ }^{4-12}$ Several consensus statements recommend different $\mathrm{PaO} 2$ and $\mathrm{SpO} 2$ thresholds to prescribe continuous positive airway pressure (CPAP),${ }^{13-15}$ non-invasive ventilation or intubation. ${ }^{16}$ Data on the association between severity of respiratory failure at admission and patients' outcomes are still limited.

The aim of the present study was to assess the clinical characteristics of patients with COVID-19 based on the severity of respiratory failure, and to explore the relationship between the degree of gas exchange impairment and clinical outcomes (CPAP initiation and mortality).

\section{METHODS}

An observational, prospective, multicentre study was conducted in three academic hospitals in Milan (Italy) from 7 March to 7 May 2020, involving three respiratory high dependency units and three general wards. A detailed list of participating centres is reported in the online supplemental file. The authors received no specific funding for this work.

\section{Patient and public involvement}

Participants were not involved in the design and conduct of the research, interpretation of results and writing of the manuscript. The results of the study will be shared with local patients' organisations by social media and summary reports on organisations' websites.

\section{Patients}

Adult hospitalised patients with a virologically confirmed diagnosis of SARS-CoV-2 infection were considered eligible for study enrolment. Patients aged $<18$ years or unable to provide informed consent were excluded from the study. Hospitalisation criteria are reported in the online supplemental file.

\section{Procedures}

Anthropometrical and clinical characteristics were collected at admission. The $\mathrm{PaO} 2 / \mathrm{FiO} 2$ ratio was calculated from the first available arterial blood gas analysis performed in the emergency department. $\mathrm{PaO} 2 /$ $\mathrm{FiO} 2$ thresholds to grade severity of respiratory failure were taken from the acute respiratory distress syndrome (ARDS) Berlin definition, and were ${ }^{17}$ : normal ( $\mathrm{PaO} 2 /$ FiO2 >300 mm Hg); mild (PaO2/FiO2 201-300 mm Hg); moderate ( $\mathrm{PaO} 2 / \mathrm{FiO} 2$ 101-200 mm Hg); severe (PaO2/ FiO2 $\leq 100 \mathrm{~mm} \mathrm{Hg}$ ). Blood count and biochemistry parameters were assessed during the first 24 hours after hospital admission.

\section{Outcomes}

The primary outcome was the description of patients' clinical characteristics at admission and the assessment of in-hospital mortality based on the severity of respiratory failure.
Secondary outcomes were the assessment of intubation rate and application of CPAP during the hospital stay.

\section{Study definitions}

SARS-CoV-2 infection and co-infections

The COVID-19 diagnosis was based on a positive nasopharyngeal swab collected in the emergency department. SARS-CoV-2 infection was proved by means of reverse transcriptase PCR (RT-PCR). In case a first swab was negative, and the clinical picture was highly suggestive for COVID-19, the swab was repeated. Co-infection with influenza virus $\mathrm{A}$ and $\mathrm{B}$, adenovirus, human rhinovirus, respiratory syncytial virus, human metapneumovirus were also investigated and analysed by means of RT-PCR or rapid influenza diagnostic tests. ${ }^{18}$ Microbiological testing for bacteria and fungi in blood, upper and lower airway tract, sputum and urinary antigens for Streptococcus pneumoniae and Legionella pneumophila were performed according to standard operating protocols.

\section{Management of respiratory failure}

Helmet CPAP was the only non-invasive respiratory support used in patients with confirmed or suspected COVID-19 pneumonia not responsive to oxygen masks in order to reduce the viral exposure of the healthcare workers in rooms without negative pressure. ${ }^{19}$ Patients with a $\mathrm{PaO} 2 / \mathrm{FiO} 2$ ratio $<300 \mathrm{~mm} \mathrm{Hg}$ in room air were administered oxygen with nasal cannulae to reach a $\mathrm{SpO} 2$ of $94 \%$ or $\mathrm{PaO} 2>60 \mathrm{~mm} \mathrm{Hg}$; in case of unsuccessful intervention within $30 \mathrm{~min}$, patients were put on reservoir masks with $90 \%-100 \%$ FiO2 or helmet CPAP was initiated with positive end expiratory pressure (PEEP) up to $12 \mathrm{cmH}_{2} \mathrm{O}$ based on the respiratory distress and comorbidities following standard operating procedures as previously described. ${ }^{14}$ CPAP failure after 2 hours with the maximal tolerable PEEP and a FiO2 of $100 \%$ was considered in case of: a) persistence of $\mathrm{PaO} 2 / \mathrm{FiO} 2<300 \mathrm{~mm}$ $\mathrm{Hg}$; b) haemodynamic instability (systolic blood pressure $<90 \mathrm{~mm} \mathrm{Hg}$ despite adequate fluid support) or altered consciousness; d) respiratory distress, fatigue and/or a respiratory rate $>30 \mathrm{bpm}^{20}$ Patients who fulfilled CPAP failure criteria were evaluated by an ICU physician for potential intubation. A do not intubate (DNI) order was established by the treating attending physician following a multidisciplinary discussion with the unit staff and the ICU and based on patient's age, comorbidities and clinical status.

\section{In-hospital treatment}

Unless contraindicated, patients received hydroxychloroquine and lopinavir/ritonavir following local standard and Italian guidelines. ${ }^{21}{ }^{22}$ In patients with severe pneumonia, methylprednisolone was given at a maximal dose of $1 \mathrm{mg} / \mathrm{kg}$ according to the American Thoracic Society/ Infectious Diseases Society of America (ATS/IDSA) guidelines $^{23}$ and local standard operating procedures. Criteria for methylprednisolone initiation included age $<80$ years, $\mathrm{PaO} 2 / \mathrm{FiO} 2<250 \mathrm{~mm} \mathrm{Hg}$, bilateral infiltrates at the chest 
X-ray or CT scan, a C reactive protein $>100 \mathrm{mg} / \mathrm{L}$ and/or a diagnosis of ARDS according to the Berlin definition. ${ }^{17}$ Immunomodulation with off-label tocilizumab at a dosage of $8 \mathrm{mg} / \mathrm{kg}$ body weight was administered in patients with signs of hyperinflammatory syndrome and elevated interleukin- $6 .{ }^{21}$ Unless contraindicated, patients received prophylactic low molecular weight heparin (LMWH) or were switched to therapeutic LMWH dosage if already on chronic anticoagulant therapy. Patients with signs of deep vein thrombosis, pulmonary embolism or D-dimer values $>5000$ received a therapeutic dose of LMWH.

\section{Statistical analysis}

Qualitative variables were summarised with absolute and relative (percentage) frequencies. Parametric and non-parametric quantitative variables were described with means (SD) and medians (IQRs), respectively. Fisher's exact and $\chi^{2}$ tests were used to compare qualitative variables, whereas Student's t-test or Mann-Whitney U test, analysis of variance or Kruskall-Wallis, corrected with Sidak adjustment, were used to compare quantitative variables with normal or non-normal distribution, respectively. Cox proportional hazard regression analysis was performed to assess the relationship between clinical outcomes and independent variables. Kaplan-Meier survival curves were plotted to show differences for the outcome mortality, considering the confounding variables age, respiratory failure, $\mathrm{PaO} 2 / \mathrm{FiO} 2$ and antihypertensive treatment; log-rank test was computed to assess the presence of any statistically significant differences. A two-tailed $p$ value $<0.05$ was considered statistically significant. All statistical computations were performed with the statistical software STATA V.16 (StatsCorp, Texas, USA).

\section{RESULTS}

\section{Clinical characteristics of the whole sample size}

A total of 412 patients were enrolled (280 males, 68\%) (table 1). The median (IQR) age at admission was 66 (55-76) years, and $54.6 \%$ of patients were $\geq 65$ years of age; $61.8 \%$ of patients had a $\mathrm{PaO} 2 / \mathrm{FiO} 2<300 \mathrm{~mm} \mathrm{Hg}$, with a median (IQR) $\mathrm{PaO} 2 / \mathrm{FiO} 2$ of 262 (140-343) mm $\mathrm{Hg}$; $24.4 \%$ had mild, $21.9 \%$ moderate and $15.5 \%$ had severe respiratory failure. CPAP was prescribed in the emergency department in $9.7 \%$ of cases, whereas only three patients were immediately intubated. Median (IQR) white blood cell (WBC) count was 6.7 (5.1-9.4) per $10^{9}$ / $\mu \mathrm{L}, 10.9 \%$ had leucopenia and $45.9 \%$ had lymphocytopenia. Median (IQR) D-dimer values were 890.5 (4702157) $\mathrm{mg} / \mathrm{L}$ fibrinogen-equivalent units (FEU) and $34 \%$ had a D-dimer $>1000 \mathrm{mg} / \mathrm{L}$ FEU (table 1 ).

Half of the patients $(50.2 \%)$ showed cardiovascular comorbidities, with hypertension being the most prevalent $(38.8 \%)$. Diabetes and chronic kidney disease were observed in $16.8 \%$ and $13.6 \%$ of the cases, respectively. Chronic obstructive pulmonary disease (COPD) and asthma accounted for the $6.1 \%$ and $3.2 \%$ of the study
Table 1 Characteristics and outcomes of patients at admission

Patients with COVID-19

( $n=412)$

\begin{tabular}{ll}
\hline Age at admission, years & $66(55-76)$ \\
\hline Males, $\mathrm{n}(\%)$ & $280(68.0)$ \\
$\begin{array}{l}\mathrm{SARS}-\mathrm{O} \text {-COV-2-positive swab, } \\
\mathrm{n}(\%)\end{array}$ & $412(100.0)$ \\
$\mathrm{PaO} 2 / \mathrm{FiO} 2$ at admission, $\mathrm{mm}$ & $262(140-343)$ \\
$\mathrm{Hg}$ &
\end{tabular}

$\mathrm{PaO} 2 / \mathrm{FiO} 2$ severity, $\mathrm{n}(\%)$

$64(15.5)$

$\leq 100 \mathrm{~mm} \mathrm{Hg}$

$\begin{array}{lc}\text { 101-200 mm Hg } & 90(21.9) \\ 201-300 ~ m m ~ H g & 101(24.4) \\ >300, \mathrm{~mm} \mathrm{Hg} & 157(38.2) \\ \text { Respiratory support at admission, } \mathrm{n}(\%) \\ \text { Room air } & 125(30.3) \\ \text { Nasal cannulae } & 93(22.6) \\ \text { Venturi mask } & 78(18.9) \\ \text { Reservoir mask } & 68(16.5) \\ \text { CPAP } & 40(9.7) \\ \text { NIV } & 5(1.2) \\ \text { IMV } & 3(0.7)\end{array}$

Blood count and biochemistry

Haemoglobin, $g / L$
$(n=401)$

Platelets, per $10^{9} / \mu \mathrm{L} \quad 203(156-270)$

$(n=401)$

$\begin{array}{lc}\begin{array}{l}\text { Platelets }<100 \text { per } 10 \% / \mu \mathrm{L}, \mathrm{n} \\ (\%) \\ (\mathrm{n}=401)\end{array} & 17(4.1) \\ \begin{array}{l}\text { White blood cells, per } 10^{9} / \mu \mathrm{L} \\ (\mathrm{n}=401)\end{array} & 6.7(5.1-9.4) \\ \text { White blood cells }<4.0 \text { per } & 45(10.9) \\ 10^{9} / \mu \mathrm{L}, \mathrm{n}(\%)(\mathrm{n}=401) & \\ \begin{array}{l}\text { Neutrophils, per } 10 \% / \mu \mathrm{L} \\ (\mathrm{n}=401)\end{array} & 5.1(3.3-8.1) \\ \begin{array}{l}\text { Neutrophils }<1.5 \text { per } 10^{9} / \mu \mathrm{L}, \\ \mathrm{n}(\%) \\ (\mathrm{n}=401)\end{array} & 7(1.7) \\ \begin{array}{l}\text { Lymphocytes, per } 10^{9} / \mu \mathrm{L} \\ (\mathrm{n}=401)\end{array} & 0.98(0.67-1.33) \\ \begin{array}{l}\text { Lymphocytes }<1.0 \text { per } 10^{9} / \\ \mu \mathrm{L}, \mathrm{n}(\%) \\ (\mathrm{n}=401)\end{array} & 189(45.9) \\ \begin{array}{l}\text { Lymphocytes }<0.5 \text { per } 10^{9} / \\ \mu \mathrm{L}, \mathrm{n}(\%) \\ (\mathrm{n}=401)\end{array} & 44(10.7) \\ \begin{array}{l}\text { Blood urea nitrogen, } \mathrm{mg} / \mathrm{dL} \\ (\mathrm{n}=372)\end{array} & 37.5(27-56) \\ \begin{array}{l}\text { Creatinine, mg/dL } \\ (\mathrm{n}=401)\end{array} & 0.93(0.75-1.19) \\ \end{array}$

Continued 
Table 1 Continued

Patients with COVID-19 $(n=412)$

\begin{tabular}{|c|c|}
\hline $\begin{array}{l}\text { Creatinine }>1.2 \mathrm{mg} / \mathrm{dL}, \mathrm{n}(\%) \\
(\mathrm{n}=401)\end{array}$ & $95(23.1)$ \\
\hline $\begin{array}{l}\text { D-dimer, mg/L FEU } \\
(\mathrm{n}=400)\end{array}$ & 890.5 (470-2157) \\
\hline $\begin{array}{l}\text { D-dimer } \geq 1000 \text { mg/L FEU, } \\
n(\%) \\
(n=195)\end{array}$ & $140(34.0)$ \\
\hline $\begin{array}{l}\text { Troponin T, ng/L } \\
(n=125)\end{array}$ & $13(7.0-22.4)$ \\
\hline $\begin{array}{l}\text { C reactive protein, } \mathrm{mg} / \mathrm{L} \\
(\mathrm{n}=400)\end{array}$ & $84.6(36.2-158.0)$ \\
\hline $\begin{array}{l}\text { Albumin, } g / L \\
(n=151)\end{array}$ & $28(23-35)$ \\
\hline $\begin{array}{l}\text { Interleukin-6 pg/mL } \\
(\mathrm{n}=83)\end{array}$ & $86(31-693)$ \\
\hline $\begin{array}{l}\text { Ferritin, } \mu g / L \\
(n=145)\end{array}$ & $1063(408-2145)$ \\
\hline
\end{tabular}

Comorbidities

Cardiovascular diseases

\begin{tabular}{lr}
$\begin{array}{l}\text { Any cardiovascular disease } \\
\mathrm{n}(\%)\end{array}$ & $207(50.2)$ \\
\hline Hypertension, $\mathrm{n}(\%)$ & $160(38.8)$ \\
Arrhythmia, $\mathrm{n}(\%)$ & $49(11.9)$ \\
$\begin{array}{l}\text { Ischaemic heart disease, } \mathrm{n} \\
\text { (\%) }\end{array}$ & $43(10.4)$
\end{tabular}

\begin{tabular}{|ll}
\hline Vasculopathy, $\mathrm{n}(\%)$ & $32(7.8)$ \\
\hline Heart failure, $\mathrm{n}(\%)$ & $17(4.1)$ \\
\hline Valvulopathy, $\mathrm{n}(\%)$ & $15(3.6)$ \\
\hline
\end{tabular}

Other

\begin{tabular}{|ll|}
\hline Diabetes mellitus, $\mathrm{n}(\%)$ & $69(16.8)$ \\
\hline Endocrinology disease, $\mathrm{n}(\%)$ & $57(13.9)$ \\
\hline Neurological disease, $\mathrm{n}(\%)$ & $49(11.9)$ \\
\hline Immune depression, $\mathrm{n}(\%)$ & $39(9.5)$ \\
\hline Hypothyroidism, $\mathrm{n}(\%)$ & $32(7.8)$ \\
\hline Kidney disease, $\mathrm{n}(\%)$ & $31(7.5)$ \\
\hline Orthopaedic disease, $\mathrm{n}(\%)$ & $31(7.5)$ \\
\hline $\begin{array}{l}\text { Gastrointestinal disease, } \mathrm{n} \\
\text { (\%) }\end{array}$ & $28(6.8)$ \\
\hline Severe obesity, $\mathrm{n}(\%)$ & $26(6.3)$ \\
\hline COPD, $\mathrm{n}(\%)$ & $25(6.1)$ \\
\hline CKD, $\mathrm{n}(\%)$ & $25(6.1)$ \\
\hline BPH, $\mathrm{n}$ (\%) & $25(6.1)$ \\
\hline Active solid cancer, $\mathrm{n}(\%)$ & $20(4.9)$ \\
\hline Previous cancer, $\mathrm{n}(\%)$ & $18(4.4)$ \\
\hline Stroke, $\mathrm{n}(\%)$ & $17(4.1)$ \\
\hline $\begin{array}{l}\text { Other neurological disease, } \\
\mathrm{n}(\%)\end{array}$ & $14(3.4)$ \\
\hline Asthma, $\mathrm{n}(\%)$ & $13(3.2)$ \\
\hline Chronic treatments & \\
\hline
\end{tabular}

Continued
Table 1 Continued

Patients with COVID-19 ( $\mathrm{n}=412)$

\begin{tabular}{|c|c|}
\hline ACEi at admission, $\mathrm{n}(\%)$ & $59(14.3)$ \\
\hline \multirow[t]{7}{*}{ ACEi name, $\mathrm{n}(\%)$} & $34(56.7)$ \\
\hline & $16(26.7)$ \\
\hline & $3(5.0)$ \\
\hline & $3(5.0)$ \\
\hline & $2(3.3)$ \\
\hline & $1(1.7)$ \\
\hline & $1(1.7)$ \\
\hline ARBs, n (\%) & $61(14.8)$ \\
\hline \multirow[t]{5}{*}{ ARB name, n (\%) } & $25(39.7)$ \\
\hline & $11(17.5)$ \\
\hline & $11(17.5)$ \\
\hline & $10(15.9)$ \\
\hline & $6(9.5)$ \\
\hline ACEi or ARBs, $n$ (\%) & $119(28.9)$ \\
\hline \multicolumn{2}{|l|}{ In-hospital treatments } \\
\hline Hydroxychloroquine, n (\%) & $336(81.6)$ \\
\hline Lopinavir/Ritonavir, n (\%) & $242(58.7)$ \\
\hline Corticosteroids, n (\%) & $105(25.5)$ \\
\hline LMWH, n (\%) & $249(60.4)$ \\
\hline Tocilizumab, n (\%) & $88(21.6)$ \\
\hline Experimental drugs, $n(\%) \dagger$ & $3(0.7)$ \\
\hline \multicolumn{2}{|l|}{ Outcomes } \\
\hline $\begin{array}{l}\text { CPAP during hospitalisation, } \\
\mathrm{n}(\%)\end{array}$ & $176(42.7)$ \\
\hline CPAP max PEEP & $10(10.0-12.5)$ \\
\hline Discharge at home, n (\%) & $180(43.7)$ \\
\hline $\begin{array}{l}\text { Discharge to other facility, } n \\
\text { (\%) }\end{array}$ & $41(10.0)$ \\
\hline In-hospital mortality, n (\%) & $105(25.5)$ \\
\hline Intubation, $\mathrm{n}(\%)$ & $36(8.7)$ \\
\hline Still hospitalised, n (\%) & $50(12.1)$ \\
\hline
\end{tabular}

Demographic, clinical characteristics, respiratory failure parameters at admission and clinical outcomes in 412 patients hospitalised with COVID-19 pneumonia. Data are expressed as frequencies or medians (IQR). Comorbidities with $\geq 3 \%$ prevalence were reported. A complete list of comorbidities is reported in table 1 of the online supplemental file. Missing values, if present, are reported next to each variable.

*At least one of the following: hypertension, arrhythmia, ischaemic heart disease, vasculopathy, heart failure, valvulopathy.

†Remdesivir.

ACEi, ACE inhibitor; ARB, angiotensin receptor blocker; $\mathrm{BPH}$, benign prostatic hyperplasia; CKD, chronic kidney disease; COPD, chronic obstructive pulmonary disease; CPAP, continuous positive airway pressure; FEU, fibrinogen-equivalent unit; IMV, invasive mechanical ventilation; LMWH, low molecular weight heparin; NIV, non-invasive ventilation; $\mathrm{PaO} 2 / \mathrm{FiO} 2$, partial pressure of oxygen to fraction of inspired oxygen ratio; PEEP, positive end expiratory pressure. 
Table 2 Patients' characteristics and outcomes depending on the severity of respiratory failure

\begin{tabular}{|c|c|c|c|c|c|}
\hline Variables & $\begin{array}{l}\text { Severe } \\
(\mathrm{PaO2} / \mathrm{FiO} 2 \leq 100 \\
\mathrm{mm} \mathrm{Hg}(\mathrm{n}=63)\end{array}$ & $\begin{array}{l}\text { Moderate } \\
\text { (PaO2/FiO2 101-200 } \\
\text { mm Hg (n=89) }\end{array}$ & $\begin{array}{l}\text { Mild } \\
\text { (PaO2/FiO2 201-300 } \\
\text { mm Hg (n=99) }\end{array}$ & $\begin{array}{l}\text { Normal } \\
\text { (PaO2/FiO2 >300 mm } \\
\mathrm{Hg}(\mathrm{n}=155)\end{array}$ & P value \\
\hline Age at admission, years & $75(64-81)$ & $72(63-81)$ & $67(57-76)$ & $58(48-70)$ & $0.0001 \dagger$ \\
\hline \multicolumn{6}{|c|}{ Respiratory support at admission, $\mathrm{n}(\%)$} \\
\hline Room air & $1(1.6)$ & $5(5.6)$ & $23(23.2)$ & $93(60.0)$ & $<0.0001 \S$ \\
\hline Venturi mask & $6(9.5)$ & $27(30.3)$ & $23(23.2)$ & $20(12.9)$ & $0.001^{\star \star}$ \\
\hline Reservoir mask & $29(46.0)$ & $31(34.8)$ & $5(5.1)$ & $3(1.9)$ & $<0.0001 \dagger \dagger$ \\
\hline CPAP & $14(22.2)$ & $9(10.1)$ & $13(13.1)$ & $4(2.6)$ & $<0.0001$ 㧊 \\
\hline NIV & $1(1.6)$ & $2(2.3)$ & $2(2.0)$ & $0(0.0)$ & 0.16 \\
\hline IMV & $1(1.6)$ & $1(1.1)$ & $1(1.0)$ & $0(0.0)$ & 0.26 \\
\hline $\begin{array}{l}\text { White blood cells, per } 10^{9} \% \\
\mu \mathrm{L}\end{array}$ & $8.3(6.2-12.2)$ & $8.1(6.0-11.0)$ & $6.5(5.1-9.0)$ & $5.9(4.8-7.7)$ & $0.0001 \S \S$ \\
\hline Neutrophils, per $10^{9} / \mu \mathrm{L}$ & $6.9(5.0-10.7)$ & $7.0(4.5-10.0)$ & $4.9(3.2-7.3)$ & $4.0(3.0-5.6)$ & 0.00019 - \\
\hline Lymphocytes, per $10^{9} / \mu \mathrm{L}$ & $0.74(0.57-0.99)$ & $0.84(0.62-1.14)$ & $1.07(0.65-1.37)$ & $1.13(0.84-1.50)$ & $0.0001^{\star \star \star}$ \\
\hline Blood urea nitrogen, mg/dL & $55(39-74)$ & $49(34-78)$ & $37(29-52)$ & $29(23-39)$ & $0.0001 \dagger † \dagger$ \\
\hline Creatinine, mg/dL & $0.91(0.8-1.3)$ & $1.04(0.76-1.39)$ & $0.92(0.74-1.15)$ & $0.89(0.72-1.05)$ & $0.007 \ddagger \ddagger \ddagger$ \\
\hline D-dimer, mg/L FEU & 1990 (701-6210) & $1355(814-4025)$ & $971(556-1830)$ & $579(336-953)$ & $0.0001 \S \S \S$ \\
\hline Troponin T, ng/L & $20(15-44)$ & $15.5(9.0-31.5)$ & $14(9-18)$ & $8(6-12)$ & 0.0001ๆๆๆ \\
\hline $\mathrm{C}$ reactive protein, $\mathrm{mg} / \mathrm{L}$ & $153(86-219)$ & $119(59-198)$ & $94.2(40.5-148)$ & $44.2(20-89.7)$ & $0.0001^{\star \star \star *}$ \\
\hline \multicolumn{6}{|l|}{ Comorbidities } \\
\hline Hypertension, n (\%) & $30(47.6)$ & $42(47.2)$ & $47(47.5)$ & $39(25.2)$ & <0.0001ๆাा \\
\hline $\begin{array}{l}\text { Ischaemic heart disease, } \\
\mathrm{n}(\%)\end{array}$ & $8(12.7)$ & $14(15.7)$ & $11(11.1)$ & $8(5.2)$ & 0.05 \\
\hline Arrythmia, n (\%) & $8(12.7)$ & $16(18.0)$ & $9(9.1)$ & $14(9.0)$ & 0.16 \\
\hline Vasculopathy, n (\%) & $8(12.7)$ & $8(9.0)$ & $9(9.1)$ & $7(4.5)$ & 0.19 \\
\hline Valvulopathy, n (\%) & $2(3.2)$ & $5(5.6)$ & $3(3.0)$ & $4(2.6)$ & 0.67 \\
\hline Heart failure, n (\%) & $3(4.8)$ & $7(7.9)$ & $4(4.0)$ & $2(1.3)$ & 0.07 \\
\hline \multicolumn{6}{|l|}{ Other } \\
\hline Diabetes mellitus, $\mathrm{n}(\%)$ & $9(14.3)$ & $21(23.6)$ & $20(20.0)$ & $18(11.6)$ & 0.07 \\
\hline $\begin{array}{l}\text { Endocrinology disease, } n \\
(\%)\end{array}$ & $7(11.1)$ & $17(19.1)$ & $13(13.1)$ & $18(11.7)$ & 0.37 \\
\hline Neurological disease, $\mathrm{n}(\%)$ & $8(12.7)$ & $16(18.0)$ & $13(13.1)$ & $12(7.7)$ & 0.12 \\
\hline Immune depression, n (\%) & $3(4.8)$ & $12(13.5)$ & $11(11.1)$ & $12(7.7)$ & 0.24 \\
\hline Hypothyroidism, n (\%) & $2(3.2)$ & $9(10.1)$ & $9(9.1)$ & $10(6.5)$ & 0.35 \\
\hline Kidney disease, n (\%) & $5(7.9)$ & $8(9.0)$ & $7(7.1)$ & $8(5.2)$ & 0.70 \\
\hline Orthopaedic disease, n (\%) & $3(4.8)$ & 7 (7.9) & $8(8.1)$ & $13(8.4)$ & 0.86 \\
\hline $\begin{array}{l}\text { Gastrointestinal disease, } \\
\mathrm{n}(\%)\end{array}$ & $6(9.5)$ & $8(9.0)$ & $4(4.0)$ & $10(6.5)$ & 0.42 \\
\hline
\end{tabular}


Table 2 Continued

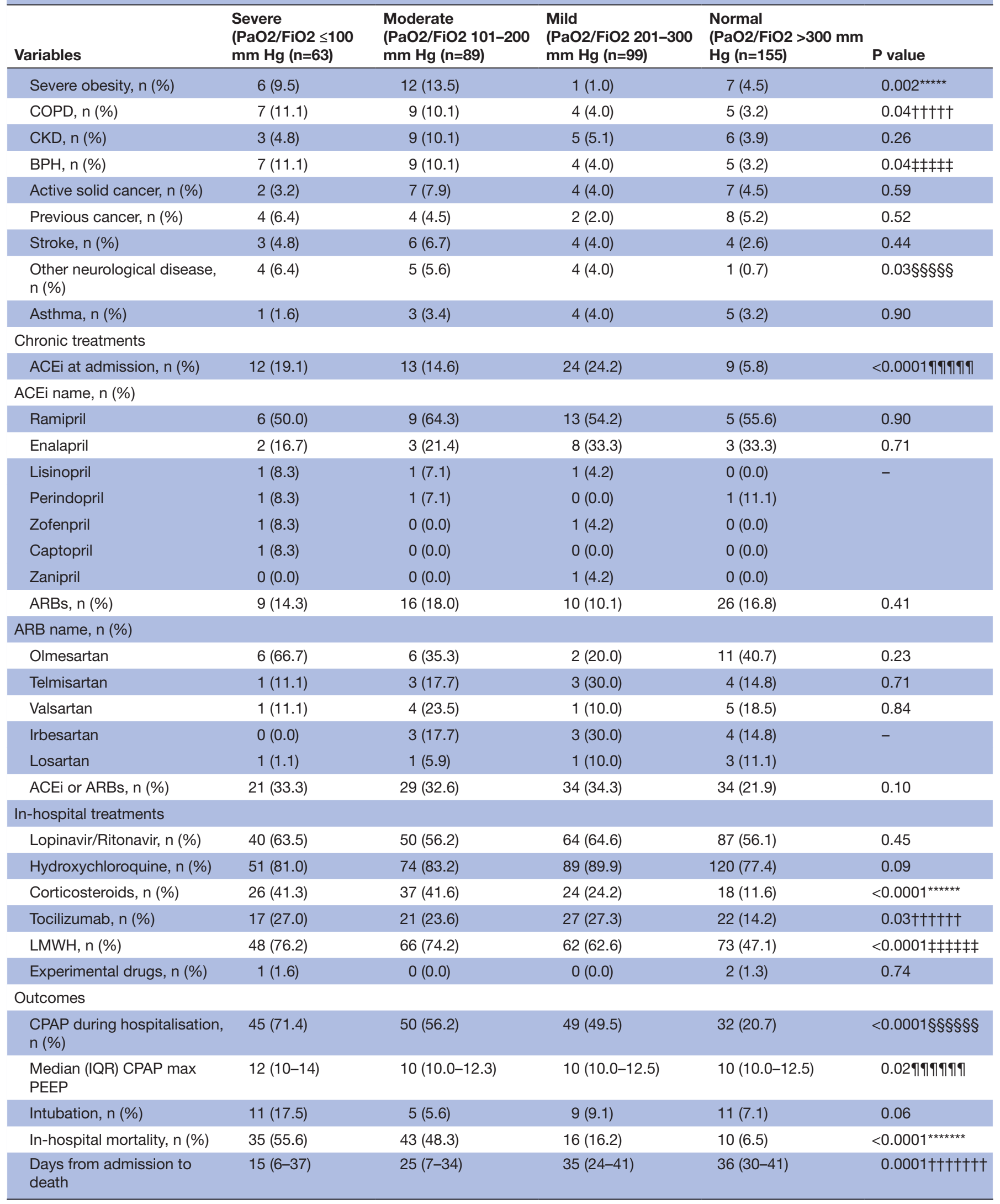

Continued 
Table 2 Continued

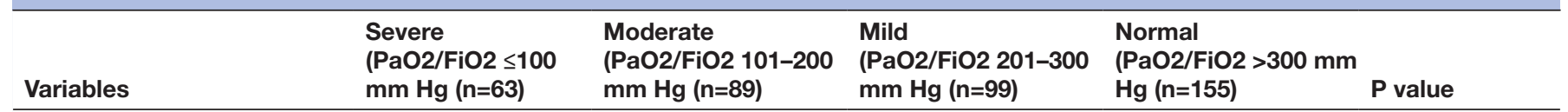

Data are expressed as frequencies or medians (IQR). Comorbidities with $\geq 3 \%$ prevalence were reported. A complete list of comorbidities is reported in table 1 of the online supplemental file.

*At least one of the following: hypertension, arrhythmia, ischaemic heart disease, vasculopathy, heart failure, valvulopathy.

†Severe vs Mild $p=0.02$; Severe vs Normal $p<0.0001$; Moderate vs Normal $p<0.0001$; Mild vs Normal $p<0.0001$.

$\ddagger$ Severe vs Mild $p=0.04$; Severe vs Normal $p=0.005$; Moderate vs Normal $p=0.03$.

$\S$ Severe vs Mild $p=0.0002$; Severe vs Normal $p<0.0001$; Moderate vs Mild $p=0.0007$; Moderate vs Normal $p<0.0001$; Mild vs Normal $p<0.0001$.

ISevere vs Mild $\mathrm{p}=0.04$; Moderate vs Mild $\mathrm{p}=0.008$.

${ }^{*}$ Severe vs Moderate $p=0.002$; Severe vs Mild $p=0.03$; Moderate vs Normal $p=0.0009$; Mild vs Normal $p=0.03$.

††Severe vs Mild $p<0.0001$; Severe vs Normal $p<0.0001$; Moderate vs Mild $p<0.0001$; Moderate vs Normal $p<0.0001$.

$\ddagger \ddagger S e v e r e$ vs Moderate $p=0.04$; Severe vs Normal $p<0.0001$; Moderate vs Normal $p=0.01$; Mild vs Normal $p=0.001$.

$\S \S S e v e r e$ vs Mild $p=0.03$; Severe vs Normal $p<0.0001$; Moderate vs Normal $p<0.0001$.

१ๆ Severe vs Mild $p=0.008$; Severe vs Normal $p<0.0001$; Moderate vs: Mild $p=0.01$; Moderate vs Normal $p<0.0001 ;$ Mild vs Normal $p=0.02$.

${ }_{* \star *}$ Severe vs Mild $p=0.01$; Severe vs Normal $p<0.0001$; Moderate vs Normal $p=0.0006$.

†††Severe vs Mild $p=0.002$; Severe vs Normal $p$-value $<0.0001$; Moderate vs: Mild $p=0.02$; Moderate vs Normal $p<0.0001 ;$ Mild vs Normal $p=0.0006$.

$\ddagger \ddagger \ddagger$ Moderate vs Normal $\mathrm{p}=0.004$.

$\S \S \S$ Severe vs Mild $p=0.02$; Severe vs Normal $p<0.0001$; Moderate vs: Mild $p=0.02$; Moderate vs Normal $p<0.0001$; Mild vs Normal $p=0.003$.

११ๆSevere vs Normal $p<0.0001$; Moderate vs: Normal $p=0.001$; Mild vs Normal $p=0.01$.

${ }_{* * * \star}$ Severe vs Mild $p=0.003$; Severe vs Normal $p<0.0001$; Moderate vs Normal $p<0.0001$; Mild vs Normal $p=0.0002$.

††† Severe vs Normal $\mathrm{p}=0.002$.

$\neq \neq \ddagger \neq$ Severe vs Normal $p=0.02$; Moderate vs: Normal $p=0.004$.

$\S \S \S \S S$ Severe vs Normal $p=0.0002$; Moderate vs Normal $p<0.0001$; Mild vs Normal $p=0.0002$.

ๆๆๆๆ|Severe vs Normal $p=0.001$; Moderate vs Normal $p=0.0004$; Mild vs Normal $p=0.0003$.

${ }_{* \star * \star *}$ Severe vs Moderate $p=0.009$; Moderate vs Mild $p=0.0007$; Moderate vs Normal $p=0.01$; Mild vs Normal $p=0.01$.

†††††Severe vs Normal $p=0.02$; Moderate vs Normal $p=0.03$.

$\ddagger \ddagger \ddagger \ddagger \ddagger$ Severe vs Normal $p=0.02$; Moderate vs Normal $p=0.03$.

$\S \S \S \S \S N A$.

ๆาๆๆी Severe vs Normal $p=0.003$; Moderate vs Normal $p=0.02$; Mild vs Normal $p<0.0001$.

$\star * \star \star \star \star$ Severe vs Mild $p=0.02$; Severe vs Normal $p<0.0001$; Moderate vs Mild $p=0.01$; Mild vs Normal $p=0.008$.

†††††Severe vs Normal $p=0.03$; Mild vs Normal $p=0.01$.

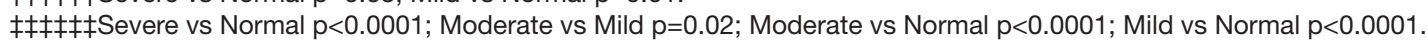

$\S \S \S \S \S \S$ Severe vs Mild $p=0.006$; Severe vs Normal $p<0.0001$; Moderate vs Normal $p<0.0001$; Mild vs Normal $p<0.0001$.

ๆๆๆๆๆๆ Severe vs Moderate $p=0.005$.

${ }_{* \star * * * * \star}$ Severe vs Mild $p<0.0001$; Severe vs Normal $p<0.0001$; Moderate vs Mild $p<0.0001$; Moderate vs Normal $p<0.0001 ;$ Mild vs Normal $p=0.01$. ††††††Severe vs Mild $p<0.0001$; Severe vs Normal $p<0.0001$; Moderate vs Normal $p<0.0001$.

ACEi, ACE inhibitor; ARB, angiotensin receptor blocker; BPH, benign prostate hypertrophy; CKD, chronic kidney disease; COPD, chronic obstructive pulmonary disease; CPAP, continuous positive airway pressure ; FEU, fibrinogen-equivalent units; IMV, invasive mechanical ventilation; LMWH, low molecular weight heparin ; NIV, non-invasive ventilation; PEEP, positive end expiratory pressure; P/F, partial pressure of oxygen to fraction of inspired oxygen ratio ( $\mathrm{PaO} 2 / \mathrm{FiO} 2)$.

sample. A complete list of observed comorbidities is reported in table 1 of the online supplemental file.

The most frequently administered therapy was hydroxychloroquine $(81.6 \%)$, whereas corticosteroids and tocilizumab were prescribed in $25.5 \%$ and $21.6 \%$ of the patients, respectively.

During the hospital stay, $42.7 \%$ were exposed to CPAP, $8.7 \%$ underwent mechanical ventilation and were transferred to the ICU.

\section{Characteristics based on severity of respiratory failure}

The cohort was divided in four groups based on the severity of respiratory failure (table 2). Advanced age and male were more prevalent in patients with severe respiratory failure ( $\mathrm{p}=0.0001$ and 0.02 , respectively).

WBC, neutrophils, $\mathrm{C}$ reactive protein and $\mathrm{D}$-dimer values were higher in severe cases (all $\mathrm{p}=0.0001$ ). Impaired gas exchange was associated with a decreased lymphocyte counts, ranging from a median (IQR) value of 1.13 (0.841.50) per $10^{9} / \mu \mathrm{L}$ in patients with $\mathrm{PaO} 2 / \mathrm{FiO} 2>300 \mathrm{~mm}$ $\mathrm{Hg}$ to $0.74(0.57-0.99)$ per $10^{9} / \mu \mathrm{L}$ in patients with severe respiratory failure $(\mathrm{p}=0.0001)$.
The proportion of patients with cardiovascular comorbidities and hypertension was significantly higher in patients with a respiratory failure if compared with that of patients with a $\mathrm{PaO} 2 / \mathrm{FiO} 2$ $>300 \mathrm{~mm} \mathrm{Hg}(\mathrm{p}<0.0001)$. Obesity was more prevalent in patients with moderate and severe respiratory failure if compared with obesity prevalence in patients with $\mathrm{PaO} 2 / \mathrm{FiO} 2 \geq 201 \mathrm{~mm} \mathrm{Hg}$ (23\% vs 5.5\%; $\mathrm{p}=0.002$ ); similar differences were found for COPD $(22.2 \%$ vs $7.2 \% ; \mathrm{p}=0.04)$. Chronic use of ACEi was more prevalent in patients with respiratory failure $(p<0.0001)$.

The highest proportion of intubated patients was in the severe group (17.5\%) (table 2 ).

\section{Impact of cardiovascular diseases and renin-angiotensin- aldosterone system inhibitors}

Overall, chronic therapy with ACEi was associated with worse $\mathrm{PaO} 2 / \mathrm{FiO} 2$ at admission (median value 223.5 vs 273.0; $\mathrm{p}=0.004$ ) (table 2 of the online supplemental file) and higher in-hospital mortality (35.6\% vs $23.5 \%$; $\mathrm{p}=0.048$ ) (table 2 of the online supplemental file and figure 1). Severity of respiratory failure at admission, intubation and mortality rates were not associated with 


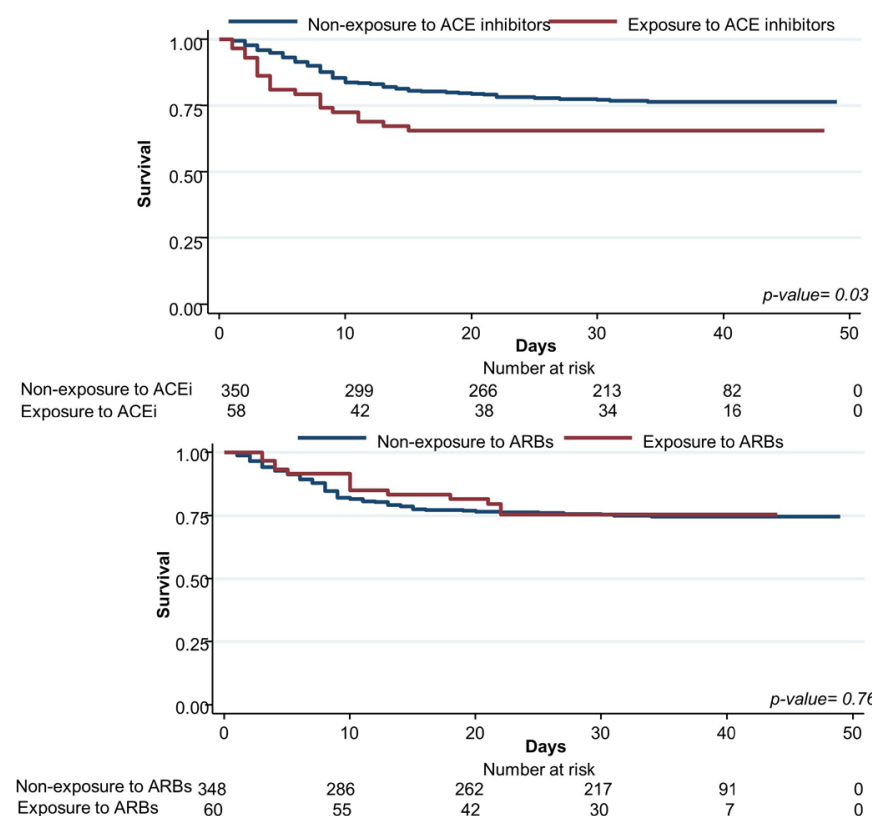

Figure 1 Survival curves based on ACE inhibitors (ACEi) or angiotensin receptor blockers (ARBs) exposure. Survival in patients hospitalised with COVID-19 pneumonia $(n=412)$ based on the chronic exposure to ACEi (upper panel) or ARBs (lower panel).

ARBs therapy (table 3 of the online supplemental file and figure 1).

Patients with CVD or hypertension had significantly lower $\mathrm{PaO} 2 / \mathrm{FiO} 2$ at admission (both $\mathrm{p}<0.0001$ ), a higher proportion of respiratory failure (both $\mathrm{p}<0.0001$ ) and an increased need for CPAP during the hospital stay $(\mathrm{p}=0.02$ and 0.003 , respectively) (table 4 of the online supplemental file and table 3 ).

\section{In-hospital mortality and respiratory failure}

In-hospital mortality was $25.5 \%$. It proportionally increased with lower $\mathrm{PaO} 2 / \mathrm{FiO} 2$ values, being highest in the severe group $(55.6 \%)$ and lowest in patients with $\mathrm{PaO} 2 / \mathrm{FiO} 2>300 \mathrm{~mm} \mathrm{Hg}(6.5 \%$; $<<0.0001)$. The number of days from admission to death was lowest in the severe group and highest in patients with normal $\mathrm{PaO} 2 / \mathrm{FiO} 2$ at admission $(\mathrm{p}=0.0001)$ (table 2). Age $>65$ years, male sex, exposure to ACEi, having a CVD, presence of respiratory failure at admission, a $\mathrm{PaO} 2 /$ FiO2 $\leq 200 \mathrm{~mm} \mathrm{Hg}$ and need for CPAP at admission were significantly associated with an increased mortality at the univariate analysis (table 4); however, the multivariate analysis showed that the only independent risk factors were age $>65$ years (HR 3.41; $95 \%$ CI 2.00 to 5.78, $\mathrm{p}<0.0001$ ), a $\mathrm{PaO} 2 / \mathrm{FiO} 2 \leq 200 \mathrm{~mm} \mathrm{Hg}$ (HR 3.57; $95 \%$ CI 2.20 to $5.77, \mathrm{p}<0.0001)$ and the presence of respiratory failure at admission (HR 3.58; 95\% CI 1.05 to $12.18, \mathrm{p}=0.04$ ) (figure 2). Fifteen days postadmission, patients with moderate-to severe respiratory failure had a survival rate of $56 \%$ (figure 2 ).

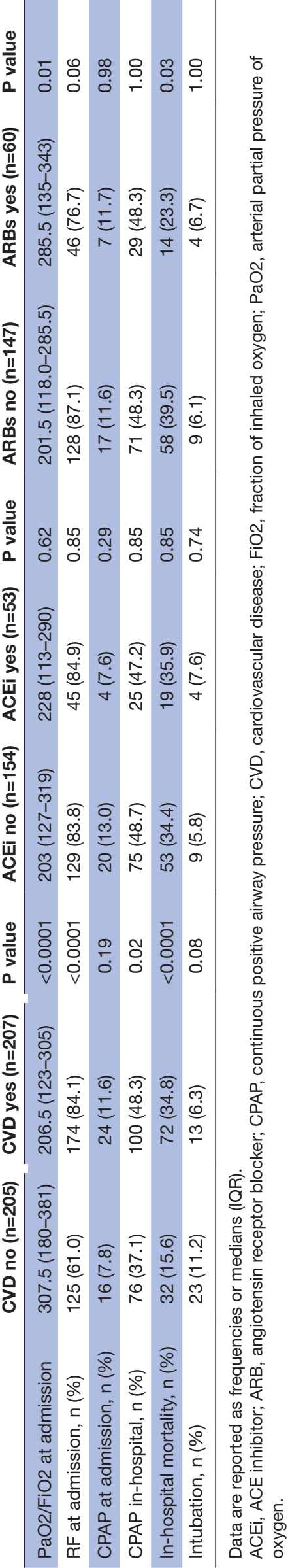


Table 4 Risk factors for in-hospital mortality

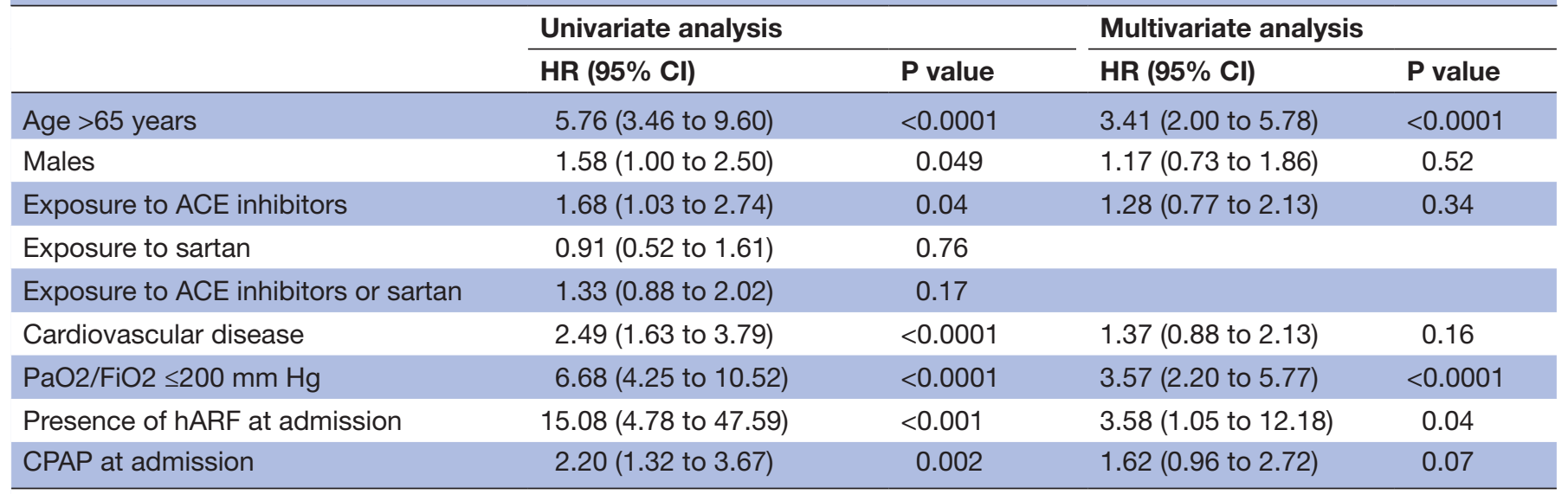

Multivariate Cox regression analysis that identifies risk factors for in-hospital mortality. Data are reported as $\mathrm{HR}$ and $95 \% \mathrm{Cls}$.

ACEi, ACE inhibitor; CPAP, continuous positive airway pressure; FiO2, fraction of inhaled oxygen; $\mathrm{PaO} 2$, arterial partial pressure of oxygen.

\section{DISCUSSION}

To the best of our knowledge, the results of the present study demonstrated for the first time the independent relationship between impaired gas exchange and clinical outcomes (mortality, intubation and need for respiratory support).

We showed that age $>65$ years, presence of respiratory failure and a $\mathrm{PaO} 2 / \mathrm{FiO} 2 \leq 200 \mathrm{~mm} \mathrm{Hg}$ at admission were independently associated with a higher mortality rate. In fact, the mortality risk for patient without respiratory failure at admission was of $1 \%$ after 15 days from hospital admission. Conversely, survival in patients with a moderate-to-severe respiratory failure $(\mathrm{PaO} 2 / \mathrm{FiO} 2$ $\leq 200 \mathrm{~mm} \mathrm{Hg}$ ) at admission was only $56 \%$ at 15 days. The overall mortality rate in our cohort is comparable to previous reports. ${ }^{524}$ However, it is higher if compared with the mortality described in other observational studies. $^{2526}$ Richardson et al reported a prevalence of respiratory failure $(\mathrm{SpO} 2<90 \%)$ of $20.4 \%,{ }^{25}$ whereas it was $72.6 \%$ in our cohort. Cheng et al reported an in-hospital mortality as low as $11 \%$ in Wuhan, China. However, $58 \%$ of enrolled patients were not discharged from hospital at the time of the report, ${ }^{26}$ whereas only $12 \%$ of our cohort was hospitalised at the time of writing.

Hypoxaemia has been rarely considered as a risk factor for patients with COVID-19. Xie et al showed that patients with $\mathrm{SpO} 2<90 \%$ had 47 times more probability to die when compared with patients with $\mathrm{SpO} 2>90 \% .{ }^{27}$ However, in patients with COVID-19-associated pneumonia, low $\mathrm{PaO} 2$ values can be associated with satisfactory SpO2, hiding hypoxia, which might lead to an underestimation of the severity of the disease and in a treatment delay. ${ }^{28}$ On this basis, clinicians should not rely solely on $\mathrm{SpO} 2$ values, especially when evaluating patients in which symptoms had lasted for 10-12 days before their presentation to the emergency department. ${ }^{29}$ The ratio between $\mathrm{PaO} 2$ and $\mathrm{FiO} 2$ has been demonstrated to be a reliable tool to assess severity and stratify mortality risk. ${ }^{17}$ When compared with the ARDS Berlin's definition, our respiratory failure classes had a slightly higher mortality with $\mathrm{PaO} 2 / \mathrm{FiO} 2<200 \mathrm{~mm} \mathrm{Hg}$ (severe $55 \%$ vs $45 \%$ and moderate $48 \%$ vs $35 \%$ ). This should probably depend on the cohort heterogeneity and in, in our case, the absence of $5 \mathrm{cmH}_{2} \mathrm{O}$ of PEEP used in the Berlin definition to grade severity of ARDS. Another issue is the low number of patients with severe respiratory failure at admission who underwent intubation $(n=11)$. This finding can be justified by the higher chance of DNI orders in patients with severe respiratory failure, secondary to the median age and to the higher prevalence of CVD. ${ }^{5}$ However, the absence of respiratory failure at admission or a mild hypoxia did not preclude the chance of in-hospital death or intubation. Sign of respiratory distress and worsening gas exchange should be closely monitored, as a sudden and rapidly evolving disease can involve patients in stable conditions. ${ }^{29} 30$

CVD and hypertension are the most frequently observed comorbidities in patients with COVID-19 and are associated with severe disease. ${ }^{31} 32$ A debate was focused on the negative effects of ACEi and ARBs due to the role of the ACE2 receptor in viral-host dynamics. ${ }^{32}$ However, several studies ruled out the increased risk of COVID-19 infection and the link between disease severity and antihypertensive treatment. $^{28} 3133$ Our cohort was characterised by a high prevalence of CVD (50.2\%), which was associated with a significantly higher mortality compared with patients without CVD. However, mortality did not change in patients chronically exposed to ACEi and ARBs. ACEi was associated with a significantly higher mortality, potentially explained by the higher disease severity of at admission of patients taking ACEi. Indeed, neither CVD, nor hypertension, nor the exposure to antihypertensive medications were independently associated with decreased survival.

\section{STUDY LIMITATIONS}

The initial gas exchange assessment was not homogeneously conducted in all patients at admission (only $30.3 \%$ of patients were in room air conditions). This might have underestimated the severity of respiratory failure, especially in patients 


\section{A}

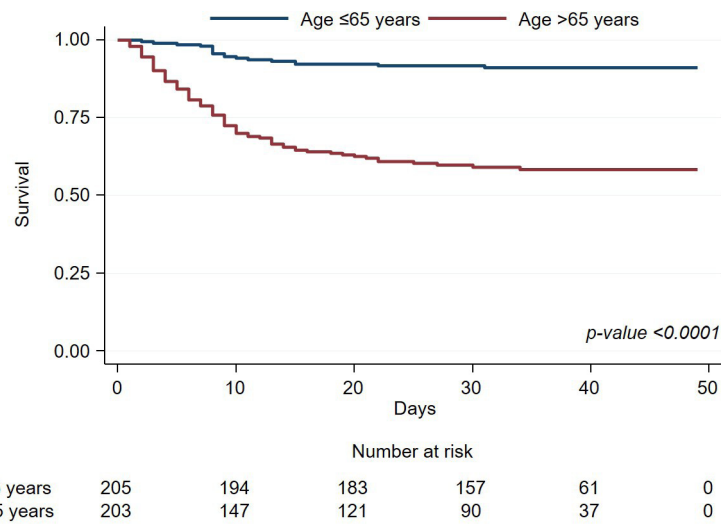

patients transferred from peripheral hospitals. The local standard operating procedures, criteria for ICU admittance or management with CPAP/NIV implemented in Italy could differ in other settings, limiting the inference of our findings.

\section{CONCLUSIONS}

The severity of respiratory failure assessed with the $\mathrm{PaO} 2 /$ $\mathrm{FiO} 2$ ratio is significantly associated with intubation rate, need for respiratory support and in-hospital mortality. Age, respiratory failure and $\mathrm{PaO} 2 / \mathrm{FiO} 2$ value at admission are independently associated with in-hospital mortality. Although the findings of the present study need to be confirmed in larger cohorts, they suggest that severity of hypoxaemia can be useful to triage patients with COVID-19 pneumonia and identify patients at higher risk of unfavourable outcomes.

\section{Author affiliations}

${ }^{1}$ Department of Biomedical and Clinical Sciences (DIBIC), Università degli Studi di Milano, Milano, Italy

${ }^{2}$ Division of Respiratory Diseases, ASST Fatebenefratelli-Sacco, Via G.B. Grassi 74-20157, Ospedale Luigi Sacco-Polo Universitario, Milano, Italy

${ }^{3}$ Dipartimento di Scienze Mediche, Chirurgiche e Sperimentali. Via Padre Manzella, 4—07100, Università degli Studi di Sassari, Sassari, Italy

${ }^{4}$ Department of Medicine and Rehabilitation, Division of Emergency Medicine, ASST Fatebenefratelli-Sacco, Piazzale Principessa Clotilde, 3-20121, 0spedale Fatebenefratelli e Oftalmico, Milano, Italy

${ }^{5}$ Division of Internal Medicine —ASST Fatebenefratelli-Sacco, Via G.B. Grassi 74-20157, 0spedale Luigi Sacco-Polo Universitario, Milano, Italy

${ }^{6}$ Direzione Sanitaria Aziendale, Via G.B. Grassi 74-20157, ASST Fatebenefratelli Sacco, Milano, Lombardia, Italy

${ }^{7}$ Department of Health Bioscience-Respiratory Unit, Policlinico di San Donato, IRCCS—Via Rodolfo Morandi, 30—20097, San Donato Milanese, Università degli Studi di Milano, Milano, Italy

${ }^{8}$ Dipartimento Medico, USC Pneumologia, USS Servizio di Pneumologia, Ospedale di Codogno, Azienda Socio Sanitaria Territoriale di Lodi, Lodi, Italy

Acknowledgements The authors would like to thank Dr Roberta Barlassina, CCP, for her help and commitment in data collection. The authors would like to thank all the colleagues, healthcare professionals and employees involved during the COVID-19 pandemic. The corresponding author here confirms that he has listed everyone who contributed significantly to the work.

Contributors PS and DR conceived the study and contributed to data collection, analysis and interpretation. GS performed the data analysis and contributed to study design and interpretation. LS performed the analysis and contributed to data interpretation. PM, CC, GDF, MR, EF, SP, FG, MDM, GN, VV and FT contributed to data collection and interpretation. PS, DR, GS and LS drafted and revised the manuscript. All authors commented on previous versions of the manuscript. All authors read and approved the final manuscript.

Funding The authors have not declared a specific grant for this research from any funding agency in the public, commercial or not-for-profit sectors.

Competing interests None declared.

Patient consent for publication Not required.

Ethics approval The study protocol (ClinicalTrials.gov: NCT04307459), designed following the amended Declaration of Helsinki (2013), was approved by the local ethical committee (Comitato Etico Milano Area I; 17263/2020) and all recruited patients gave written informed consent.

Provenance and peer review Not commissioned; externally peer reviewed.

Data availability statement PS and DR had full access to all the data in the study and take responsibility for the integrity of the data and the accuracy of the data analysis and had final responsibility for the decision to submit for publication. The datasets used and analysed during the current study are available from the corresponding author on reasonable request. 
Supplemental material This content has been supplied by the author(s). It has not been vetted by BMJ Publishing Group Limited (BMJ) and may not have been peer-reviewed. Any opinions or recommendations discussed are solely those of the author(s) and are not endorsed by BMJ. BMJ disclaims all liability and responsibility arising from any reliance placed on the content. Where the content includes any translated material, BMJ does not warrant the accuracy and reliability of the translations (including but not limited to local regulations, clinical guidelines, terminology, drug names and drug dosages), and is not responsible for any error and/or omissions arising from translation and adaptation or otherwise.

Open access This is an open access article distributed in accordance with the Creative Commons Attribution Non Commercial (CC BY-NC 4.0) license, which permits others to distribute, remix, adapt, build upon this work non-commercially, and license their derivative works on different terms, provided the original work is properly cited, appropriate credit is given, any changes made indicated, and the use is non-commercial. See: http://creativecommons.org/licenses/by-nc/4.0/.

ORCID iD

Pierachille Santus http://orcid.org/0000-0003-3462-8253

\section{REFERENCES}

1 World Health Organization. COVID-19 situation report - 197, 2020. Available: https://www.who.int/docs/default-source/coronaviruse/ situation-reports/20200804-covid-19-sitrep-197.pdf?sfvrsn= 94f7a01d_2

2 Marini JJ, Gattinoni L. Management of COVID-19 respiratory distress. JAMA 2020;323:2329-30.

3 Carsana L, Sonzogni A, Nasr A, et al. Pulmonary post-mortem findings in a large series of COVID-19 cases from northern Italy. Lancet Infect Dis 2020;S1473-3099:30434-5.

4 Zhou F, Yu T, Du R, et al. Clinical course and risk factors for mortality of adult inpatients with COVID-19 in Wuhan, China: a retrospective cohort study. Lancet 2020;395:1054-62.

5 Grasselli G, Zangrillo A, Zanella A, et al. Baseline characteristics and outcomes of 1591 patients infected with SARS-CoV-2 admitted to ICUs of the Lombardy region, Italy. JAMA 2020;323:1574-81.

6 Du R-H, Liang L-R, Yang C-Q, et al. Predictors of mortality for patients with COVID-19 pneumonia caused by SARS-CoV-2: a prospective cohort study. Eur Respir J 2020;55:2000524.

7 Yang X, Yu Y, Xu J, et al. Clinical course and outcomes of critically ill patients with SARS-CoV-2 pneumonia in Wuhan, China: a singlecentered, retrospective, observational study. Lancet Respir Med 2020;8:475-81.

$8 \mathrm{Li}$ J, Wang X, Chen J, et al. Association of renin-angiotensin system inhibitors with severity or risk of death in patients with hypertension hospitalized for coronavirus disease 2019 (COVID-19) infection in Wuhan, China. JAMA Cardiol 2020;5:825-6.

9 World Health Organization. Clinical management of severe acute respiratory infection (SARI) when COVID-19 disease is suspected, 2020. Available: https://www.who.int/publications/i/item/clinicalmanagement-of-covid-19 [Accessed 5 Aug 2020].

10 China CDC Weekly. The epidemiological characteristics of an outbreak of 2019 novel coronavirus diseases (COVID-19) - China, 2020. China CDC Weekly 2020;2:113-22.

$11 \mathrm{Hu}$ L, Chen S, Fu Y, et al. Risk factors associated with clinical outcomes in 323 COVID-19 hospitalized patients in Wuhan, China. Clin Infect Dis 2020:ciaa539.

12 Feng Y, Ling Y, Bai T, et al. COVID-19 with different severities: a multicenter study of clinical features. Am J Respir Crit Care Med 2020;201:1380-8.

13 Vitacca M, Nava S, Santus P, et al. Early consensus management for non-ICU acute respiratory failure SARS-CoV-2 emergency in Italy: from ward to trenches. Eur Respir J 2020;55:2000632.
14 Radovanovic D, Rizzi M, Pini S, et al. Helmet CPAP to treat acute hypoxemic respiratory failure in patients with COVID-19: a management strategy proposal. J Clin Med 2020;9:1191.

15 Winck JC, Ambrosino N. COVID-19 pandemic and non invasive respiratory management: every Goliath needs a David. An evidence based evaluation of problems. Pulmonology 2020;26:213-20.

16 Phua J, Weng L, Ling L, et al. Intensive care management of coronavirus disease 2019 (COVID-19): challenges and recommendations. Lancet Respir Med 2020;8:506-17.

17 Ranieri VM, Rubenfeld GD, Thompson BT, et al. Ards definition Task force. acute respiratory distress syndrome: the Berlin definition. JAMA 2012;307:2526-33.

18 Radovanovic D, Sotgiu G, Jankovic M, et al. An international perspective on hospitalized patients with viral community-acquired pneumonia. Eur J Intern Med 2019;60:54-70.

19 Ferioli M, Cisternino C, Leo V, et al. Protecting healthcare workers from SARS-CoV-2 infection: practical indications. Eur Respir Rev 2020;29:200068.

20 Aliberti S, Radovanovic D, Billi F, et al. Helmet CPAP treatment in patients with COVID-19 pneumonia: a multicenter, cohort study. Eur Respir J 2020:2001935.

21 SIMIT. Societ Italiana di Malattie Infettive e Tropicali. In: Sezione Regione Lombardia. Vademecum per La cura delle persone Con malattia dA COVI-19. 2.0 ed, 2020. http://www.simit.org/medias/ 1569-covid19-vademecum-13-03-202.pdf

22 Bassetti M, Giacobbe DR, Aliberti S, et al. Italian Society of antiinfective therapy (SITA) and the Italian Society of pulmonology (SIP). balancing evidence and frontline experience in the early phases of the COVID-19 pandemic: current position of the Italian Society of anti-infective therapy (SITA) and the Italian Society of pulmonology (SIP). Clin Microbiol Infect 2020;26:880-94.

23 Metlay JP, Waterer GW, Long AC, et al. Diagnosis and treatment of adults with community-acquired pneumonia. An official clinical practice guideline of the American thoracic Society and infectious diseases Society of America. Am J Respir Crit Care Med 2019;200:e45-67.

24 Guan W-J, Ni Z-Y, Hu Y, et al. Clinical characteristics of coronavirus disease 2019 in China. N Engl J Med 2020;382:1708-20.

25 Richardson S, Hirsch JS, Narasimhan M, et al. Presenting characteristics, comorbidities, and outcomes among 5700 patients hospitalized with COVID-19 in the new York City area. JAMA 2020;323:2052-9.

26 Chen N, Zhou M, Dong X, et al. Epidemiological and clinical characteristics of 99 cases of 2019 novel coronavirus pneumonia in Wuhan, China: a descriptive study. Lancet 2020;395:507-13.

27 Xie J, Covassin N, Fan Z, et al. Association between hypoxemia and mortality in patients with COVID-19. Mayo Clin Proc 2020;95:1138-47.

28 Tobin MJ, Laghi F, Jubran A. Why COVID-19 silent hypoxemia is Baffling to physicians. Am J Respir Crit Care Med 2020;202:356-60.

29 Tobin MJ. Basing respiratory management of COVID-19 on physiological principles. Am J Respir Crit Care Med 2020;201:1319-20.

30 Gandhi RT, Lynch JB, del Rio C. Mild or moderate Covid-19. N Engl J Med Overseas Ed 2020.

31 Reynolds HR, Adhikari S, Pulgarin C, et al. Renin-AngiotensinAldosterone system inhibitors and risk of Covid-19. N Engl J Med 2020;382:2441-8.

32 Gupta AK, Jneid H, Addison D, et al. Current Perspectives on Coronavirus Disease 2019 and Cardiovascular Disease: A White Paper by the JAHA Editors. J Am Heart Assoc 2020;9:e017013.

33 Mancia G, Rea F, Ludergnani M, et al. Renin-AngiotensinAldosterone system blockers and the risk of Covid-19. N Engl J Med 2020;382:2431-40. 